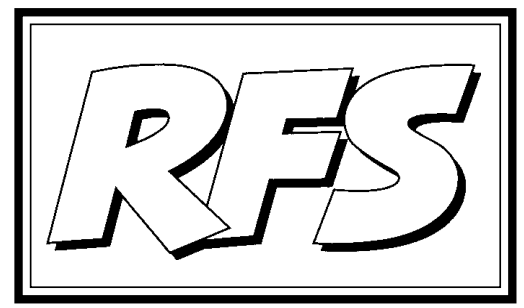

Revista de Fomento Social, 53 (1998), 119-130

\title{
La Mujer y el Mercado Laboral: Una especial referencia a los puestos directivos
}

Es una realidad en la sociedad actual la discriminación de la mujer en el mercado laboral por unas $u$ otras causas. La autora presenta un acercamiento a esta realidad desde un punto de vista teórico, y a su vez cercano concretando en la participación de la mujer en los puestos directivos. Describe las limitaciones de la mujer y las aportaciones que puede hacer ésta al mercado laboral.

$(*)$

(*) Profesora de la Facultad de Ciencias Económicas y Empresariales - ETEA, de la Universidad de Córdoba y Gerente de ETEA. 


\section{Introducción}

El problema de la incorporación de la mujer a los puestos directivos se enmarca en la problemática general de la discriminación de la mujer en el mercado laboral, por ello, se empezará por analizar cuál es la realidad en cuanto a la incorporación de la mujer al empleo, y cuáles son las causas que marcan esta realidad. Se analizarán los condicionantes que señalan la actuación de la mujer en el mercado laboral, y se finalizará con una revisión de valores o aptitudes que tiene la mujer y que pueden aportar en su trabajo, desde un punto de vista general, y específicos para el caso de que ocupe un puesto directivo.

\section{1. ¿Cuál es la realidad y por qué se ha llegado a ella?}

Si se analiza la información estadística existente sobre el mercado laboral, comparando ambos sexos, encontramos discriminaciones en distintos aspectos de la mujer respecto del hombre:

En lo referente al acceso al puesto de trabajo, con una formación similar, las mujeres tienen menos opciones para encontrar un empleo adecuado a su formación que los hombres. Así, según datos de junio de 1997, mientras que la tasa de desempleo de los hombres era de un 16,07 por ciento, la de las mujeres alcanzaba el 28,35 por ciento. En ese mismo mes, de los 59.200 nuevos puestos de trabajo creados, sólo 8.900 fueron ocupados por mujeres. En este sentido, España es mucho más discriminante que la UE, ya que en ésta se dan diferencias de tasa de desempleo tan sólo en torno a un dos por ciento entre la masculina y la femenina.

Estas diferencias corroboran que las reivindicaciones de las mujeres europeas en la IV Conferencia Mundial sobre la Situación de la Mujer en el Mundo celebrada en 1995, se centraran en el hecho de que hay que fomentar el papel de la mujer, incrementando el número de cargos políticos y decisorios. Pero en España la reivindicación tiene que empezar por la igualdad en el acceso al mercado laboral. En todo caso, en España se vive una situación de privilegio, ya que la reivindicación de muchos países comienza por la igualdad en la educación.

Esta situación de discriminación de la mujer en el mercado laboral se presenta a pesar de estar demostrado que las mujeres de la UE logran mejores resultados en los niveles de educación, y consiguen mayores resultados en el desarrollo de 
su trabajo que los hombres, sin embargo, van por detrás de los hombres en su lucha por encontrar un empleo.

El sector de la actividad en el que se incorporan viene marcado por los estudios que realizan. En general, las mujeres optan por las titulaciones de letras y medicina, incluyendo enfermería, mientras que los hombres se dirigen hacia las ciencias, matemáticas, informática, ingeniería y arquitectura. De hecho, según la encuesta de población activa del tercer trimestre de 1997, clasificada por ramas de actividad, el número de mujeres es casi el doble o más respecto al de los hombres en: educación, actividades sanitarias, servicios sociales, en lo que se denomina otras actividades (como pueden ser el servicio doméstico), así como en parados que buscan el primer empleo o que han dejado su último empleo hace tres años o más, entre los que se encuentra lo que llamamos economía sumergida.

Asimismo, existen diferencias cualitativas, puesto que las mujeres siguen ocupando empleos de menor cualificación y remuneración, de hecho tan sólo ocupan el 18 por ciento de los trabajos mejor pagados, frente al 82 por ciento acaparados por los hombres. Se da la paradoja de que si un mismo puesto de trabajo es infravalorado en un país, éste es ocupado mayoritariamente por mujeres, y si es muy valorado en otro país, entonces es ocupado mayoritariamente por hombres.

En cuanto a la ocupación de puestos directivos, la presencia femenina en las direcciones generales es todavía escasa, pero en los escalones jerárquicos intermedios se aprecia un rápido aumento en la proporción de directivas. Así, las mujeres ocupan un 8 por ciento de los puestos de los comités de dirección, se habla que ocupan entre un 10 y un $15 \%$ de los puestos directivos, y en concreto, su presencia es entre un 20 y un 30 por ciento en los departamentos de Marketing y Recursos Humanos. Desde principios de esta década se ha apreciado un incremento de aproximadamente un 20 por ciento en la proporción femenina en los puestos de dirección.

En cuanto a la posibilidad de trabajar como profesional, o de ejercer como empresarias, la mujer se muestra recelosa. Según el Boletín de Estadísticas Laborales de las mujeres ocupadas en el tercer trimestre de 1997, tan sólo un $15 \%$ de las mujeres ocupadas está ejerciendo como empresaria o autónoma, frente al $23 \%$ de los hombres ocupados. Sin embargo, se ha de destacar, que analizando esta información en el contexto de la relativa reciente incorporación de la mujer al mercado laboral, parece que no existen grandes diferencias entre 
ambos sexos. En realidad esta posibilidad se está presentando como una vía de salida para aquellas mujeres que encuentran limitaciones en encontrar un trabajo por cuenta ajena.

En cuanto a la definición de las causas que han llevado a esta realidad, existen diferentes estudios que han analizado el cambio estructural en el mercado de trabajo por la incorporación de la mujer al mercado laboral, así como sobre las razones que impulsan a la mujer a formar parte de este mundo (1).

Estos estudios concluyen que la terciarización de la economía, es decir, el predominio del sector servicios sobre la agricultura y la industria en la economía española, y el desarrollo tecnológico, se han traducido en una mayor proporción del trabajo no manual, lo que ha beneficiado especialmente a la mujer. Así, según la encuesta de población activa en el tercer trimestre de 1997, el 70 por cien de las mujeres empleadas desempeñan un trabajo no manual frente a un porcentaje del $43 \%$ para los hombres.

Además, el mayor nivel de educación de la mujer y el descenso en el número de hijos ha permitido, en gran medida, su acceso al mercado laboral. En relación al descenso del número de hijos, hay que resaltar que este descenso no es sólo consecuencia de la incorporación de la mujer al trabajo, sino de la nueva estructura familiar y del coste económico que supone tener un hijo, es decir, la decisión de tener menos hijos es previa, en algunos casos, a la de la búsqueda de trabajo, y lo que hace es facilitar estaúltima decisión. El mayor nivel de educación supone que acceden, en mayor medida, a los empleos cualificados que a los que no necesitan especialización.

Otro factor que también influye, aunque en menor medida, es el ciclo económico. Cuando la economía está en auge aumenta la probabilidad de encontrar empleo, lo que incentiva a entrar en el mercado de trabajo.

Por último, otro de los aspectos que destacan los distintos estudios como influyentes en el aumento de la participación femenina en el mundo laboral es la «precarización laboral», es decir, el deterioro de la calidad de los empleos: menor estabilidad laboral y menores salarios. Ello ha permitido crear un hueco en el mundo laboral para la mujer, ya que a los responsables de las empresas se les presenta la posibilidad de no arriesgar mucho al contratar una mujer si el

(1) Bover, Olimpia, «Cambios en la composición del empleo y actividad laboral femenina» (1976-1992). Estudio del Banco de España. 
contrato no es indefinido o si puede pagarle menos que a un hombre. Las mujeres tienen mucha menos familiaridad que los hombres con la regulación del mercado de trabajo, y por ello, son mucho más proclives a admitir condiciones de trabajo menos favorables. De hecho, una vez inmersas en el mundo laboral, encontramos discriminación en el tipo de contrato ofrecido a la mujer. Así, según el Boletín de Estadísticas Laborales en el último trimestre de 1996 un 36 \% de las mujeres tienen contratos temporales, frente al 28 por cien de los hombres, y en cuanto a la dedicación, un $16 \%$ de las mujeres tienen contratos a dedicación parcial frente al 3,8\% de los hombres. Cuando se piensa en apoyar los contratos a dedicación parcial se señala lo especialmente indicados que son para los jóvenes y para las mujeres. Es verdad, que son especialmente indicados para facilitar la incorporación al trabajo de la mujer que por sus circunstancias personales le impiden desarrollar una jornada completa, o porque así se ha planteado su vida personal, pero no debe considerarse así como consecuencia de que se conciba el trabajo de la mujer como un complemento del trabajo principal o, como piensan algunos, para obtener una retribución cuyo destino es ropa y caprichos. El contrato parcial debe ser una opción no una imposición.

\section{Condicionantes en la actuación de la mujer en el mercado laboral}

En este apartado se va a analizar una serie de variables o aspectos que influyen en el desarrollo de la mujer en su trabajo, y que por tanto, afectan a cualquier tipo de mujer, ocupe el puesto que ocupe.

- Maternidad: La maternidad hace de las mujeres sujetos con incapacidades temporales para trabajar durante el periodo de gestación, el parto y la lactancia, con limitaciones para desempeñar ciertas actividades que puedan dañar a su hijo, y propensas a adquirir malestares y enfermedades específicos (2). Estas circunstancias han sido reconocidas por la legislación laboral, pero no aceptadas por la sociedad, las empresas se sienten perjudicadas por estos hechos, por las implicaciones que conllevan, ya sea abandono temporal de una determinada tarea, sustitución de una persona, disminución de la jornada laboral, etc. No es raro encontrarse con mujeres

(2) RENDÓN, Teresa, «El trabajo femenino en el mundo». Sistema, nº140-141, noviembre, 1997,pp.139-154. 
a las que no se les ha renovado el contrato cuando se han quedado embarazadas, o que no se piense en ellas para ocupar una serie de cargos si están en la edad propia de tener hijos.

- La convivencia de los tres papeles de la mujer: madre, esposa y trabajadora. La tensión existente entre estos tres papeles se hace más intensa a medida que la mujer tiene mayor responsabilidad en su trabajo por el mayor tiempo y dedicación que le requiere.

En la familia o en el hogar, a pesar de que las nuevas generaciones de hombres entienden que es tarea de todos la responsabilidad del hogar, sin embargo, no están educados para ello, es la mujer recién casada la que enseña a su marido el trabajo doméstico, pero con ello no consigue el compartir la responsabilidad. Hay numerosos estudios sobre la dedicación del hombre y la mujer a las actividades del hogar, en el caso de que la mujer trabaje fuera del hogar, que demuestran que existen grandes diferencias entre la dedicación de la mujer y la del hombre, que además se traducen en una concentración de la realización de las actividades en los fines de semana tanto en el hombre como en la mujer (3), pero presentándose en ésta con mayor intensidad, y que la distribución del trabajo se hace de forma más asimétrica en las tareas propiamente domésticas que en las actividades relacionadas con la adquisición de bienes y servicios, y del cuidado de los niños.

El hecho es, que es responsabilidad de la mujer hasta el más mínimo detalle, aunque no ejecute las distintas tareas porque tenga ayuda, tiene que encargarse de pensarlas y prever los distintos aspectos para su ejecución. Cuántas veces se ha dicho que lo que más disgusta no es el hacer la comida sino tener que pensar en lo que se va a poner de comer.

Como madre, la mujer comienza por sentirse desplazada cuando los niños son pequeños y los tiene que dejar al cuidado de otra persona, ¿quién no ha sentido envidia de la cuidadora en los primeros meses del bebé?, sobre todo si encima se trata de un familiar. Después, cuando los niños tienen uso de razón y empiezan a tener sus propias actividades, se siente pánico de lo que se está perdiendo, entra el complejo de culpabilidad de si no se está

(3) Un estudio de la desigualdad en el uso del tiempo del domingo, se puede consultar en CAllejo, J., «Trabajadoras del domingo», Revista Fomento Social, n 207, vol. 52, pp. 369-386. 
disponible siempre que debes.

Como esposa, antes, la mujer era una fiel sirvienta de su marido, vivía para sus hijos y para él. Hoy esta relación ha cambiado, se pretende que la pareja sea unión, el unopara el otroen todos los sentidos. El hombre tieneque entenderque los distintos papeles que tiene la mujer actual le hace viviren un estado detensión que implica que no puede ser todo dar, también necesita recibir.

Pese a todo lo anterior, todavía, la mayoría de las veces, es la mujer la que tiene que ceder en su vida profesional para que el hombre no se vea interrumpido en su carrera. Existen infinidad de casos en los que la mujer ha tenido que abandonar su puesto de trabajo por ser incompatible con el de su marido, o por necesidades de su familia; esto no se ve mal si se toma esta decisión desde la libertad y desde el convencimiento de que eso es lo que quieres, pero no como norma.

Cuando se da el éxito profesional, en muchos casos, se debe a que éste es compatible con la vida profesional del marido o, en otros casos, ocasiona el destrozo de la vida familiar.

Si además coincide que el matrimonio trabaja en una empresa familiar, generalmente es la mujer la que tiene que buscar la frontera entre la vida privada y la convivencia profesional, es la mujer la que lucha por buscar un tiempo y un espacio propios para la pareja y para la familia.

Las mujeres de hoy no han sido educadas para quedarse limitadas, se ha luchado por conseguir un sitio en el mercado laboral y no se quieren quedar cojas en ninguno de los sentidos, quieren que se les ayude a encontrar el equilibrio en estos tres papeles: madre, esposa y trabajadora.

- Cuidado de las personas mayores: Otra tarea tradicionalmente asignada a las mujeres es el cuidado de las personas mayores, lo que supone una responsabilidad añadida a la comentada en el apartado anterior, que conlleva una limitación más de la mujer, y a veces provoca incluso el abandono temporal del puesto de trabajo si no encuentra una ayuda que no sea muy costosa.

- Familias monoparentales: Si todo lo anterior dificulta la actuación de la mujer, se agrava en el caso de que ésta sea la única que encabeza la familia, madres solteras, separadas o divorciadas, y viudas. A los problemas que se ha hecho referencia anteriormente se ha de añadir la dificultad de incorporarse al mercado laboral después de estar años dedicados a la vida doméstica. 
- Visibilidad (4): La mujer se encuentra en situaciones (reuniones o incluso en su trabajo) en las que es la única persona del sexo femenino. Este hecho hace que sea el foco de atención, que su actuación sea juzgada, analizada y comentada. Este análisis se hace en ocasiones bajo una perspectiva influida por los estereotipos existentes sobre la mujer en general. ¿Cuántas veces se ha oído que las mujeres son todas unas histéricas y que su corazón se adueña de la cabeza?. En muchas ocasiones los problemas que surgen en la empresa se asocian a la visión estereotipada de la mujer, sin buscar las verdaderas causas, con el consiguiente perjuicio que ello conlleva de cara a una futura contratación. No todas las mujeres son iguales, así como no todos los hombres son iguales. Cada persona tiene su singularidad.

- Rendimiento: El análisis que se hace de su trabajo viene marcado por una escala distinta a la de sus compañeros.

De por sí, ya ha tenido que ser mejor que sus rivales a la hora de acceder al puesto de trabajo, ya que entre un hombre y una mujer exactamente iguales en una valoración se va a seleccionar al hombre. Por tanto, la mujer tiene que ser consciente de que para su formación debe adquirir complementos que mejoren sucurriculum.

En cuanto a su promoción, también su progresión debe ser más espectacular que la de sus compañeros para que se vea reflejada en ascensos. Todo esto crea en la mujer un instinto de continua superación que, por un lado, es un estado continuo de tensión, y por otro, de complacencia una vez que supera sus retos.

- Desintegración: Se mueve en un ambiente que todavía está programado por las reglas de los hombres. Las normas de comportamiento del equipo de trabajo son las masculinas, y ello provoca que la mujer tenga que ir continuamente adaptándose e incluso intentando cambiar ese entorno. Por ejemplo, a la hora de fijar las reuniones, para los hombres nunca hay problema en fijarla incluso en un día no laborable, y la mujer se tiene que adaptar a este comportamiento a pesar de que es contraria a ello, por no ser ella la que se opone.

- Infravaloración: Las limitaciones que la sociedad pone a la mujer han ido

(4) Amanent, Rosa $\mathrm{M}^{\mathrm{a}}$., «La perspectiva de la mujer ejecutiva en la empresa». Alta Dirección, nº121, 1985. 
generando un sentimiento de inferioridad en ella, que en muchos casos hace que no se crea capaz de acceder al mercado laboral y, en otros, que se considere muy inferior a sus compañeros. Para evitar esto, es necesario ponerse pequeños retos cuya superación irá proporcionando seguridad en sí misma. La causa de que en algunas ocasiones se promocione a un hombre frente a una mujer es que la empresa no es consciente de los valores de ésta, la mujer no hace ver su cualificación porque se cree inferior a su compañero, a pesar de que esté más formada y sea mucho más flexible.

- Mutilación en el desarrollo personal: La doble jornada de la mujer redunda en limitaciones en distintos aspectos de su desarrollo personal, como son el aspecto cultural, la imposibilidad de desarrollo profesional, la imposibilidad de relacionarse con los demás, o de ocio.

La mujer ha tenido que asumir una concepción del tiempo hecha por y para el hombre, que se basa en la primacía del tiempo de trabajo remunerado sobre los otros tiempos, tales como el dedicado al hogar, el dedicado a los demás o el dedicado a sí misma.

Si se necesita hacer un curso que vendría bien para promocionarse, no se tiene tiempo después de la jornada laboral porque se le quitaría al tiempo que se dedica a la familia.

No se tiene tiempo ni de leer el periódico o de leer un libro, salvo en vacaciones. Si sobra algo de tiempo siempre hay algo pendiente de leer en relación con el trabajo.

Cuántas veces se ha echado de menos el poder estar tranquilamente charlando con algún amigo/a.

La falta de tiempo impide hacer cosas que gustan, o no disfrutar de lo que se hace. Si gustaba cocinar, ahora no se disfruta de ello.

\section{Aptitudes que tiene la mujer y que puede aportar al mercado laboral.}

En la actualidad se está produciendo una evolución de las estructuras organizativas de la empresa, pasando de una organización jerárquica basada en el predominio de los valores considerados «masculinos»: racionalidad, energía, durezay capacidad analítica, a una organización en la que son necesarios valores como la afectividad, la sensibilidad, lacreatividad, el detalle. Rasgostodosellos propiamente «femeninos», por lo que las mujeres tienen mucho que aportar a las nuevas formas de dirección. 
Por ello se va a revisar de forma muy somera algunos de estos valores que tiene la mujer y que pueden tener un efecto positivo en las organizaciones, efecto que será mayor cuanto mayor sea la responsabilidad de la mujer.

La responsabilidad de la mujer en la familia proporciona una experiencia a considerar en la valoración de la mujer en el mundo laboral: una empresa es un ente similar a una familia, la responsabilidad recae de igual forma en: las instalaciones, la tarea diaria y el personal, es decir, la casa, las labores diarias y los componentes de la familia, hijos y esposo. Si a los conocimientos domésticos que tiene para llevar adelante una familia se le une la formación necesaria para el desarrollo del trabajo, será totalmente capaz de dirigir.

Todavía hoy, la mujer es educada para que sea sencilla y poco vanidosa, ello se refleja en que es capaz de reconocer sus propios errores y dar marcha atrás si es necesario. Cualidad que es esencial en puestos de alta responsabilidad.

La mujer es más sensible en todos los aspectos, ello facilita las relaciones humanas dentro y fuera de la empresa. La mujer no olvida que es solamente un ser humano, y ello hace que acepte su situación. Una de las reglas de juego más normal entre los hombres es la agresividad en la actividad laboral, la imposibilidad de expresar emociones sin que le acusen de debilidad, «si te daba un ataque de nervios eres un fracasado». Sin embargo, a la mujer no le importa hablar de sus sentimientos, no le importa hablar de los hijos en un momento determinado, y que ello quite frialdad y rigidez en los contactos de negocios, mientras que a muchos hombres ello les resulta sensiblero y piensan que ese hecho les puede arrebatar la imagen de dureza que quieren dar.

Por otro lado, esa mayor sensibilidad de las mujeres facilita la comunicación en todos los sentidos. Generalmente, la mujer tiene más capacidad para escuchar y le da mayor importancia a este aspecto, por lo que se esfuerza en ser más accesible, sobre todo con los subordinados más inmediatos.

La continua lucha de la mujer por hacerse respetar le ha llevado a tener como principio prioritario «el respeto a los demás», que a su vez se manifiesta en la aplicación de un principio de igualdad dentro de la empresa en todos los sentidos.

La mujer es más polifacética, es más capaz de hacer el papel que corresponde en cada momento. Esto le facilita la ejecución de las tareas propias de puestos directivos en el entorno actual tan cambiante, en donde es muy importante la adaptabilidad de las personas.

El hombre ha sido educado para el logro, por ello suele ser organizado, 
planificado; sin embargo, la mujer ha sido educada para dar, para enfrentarse a cualquier situación en cualquier momento, y ante ello cabe cualquier contrariedad y la necesidad de ir marcando prioridades. A la mujer le preocupa más el proceso que el fin, se preocupa más por los efectos que pueda tener cualquier decisión, mientras que en el hombre prevalece la consecución del objetivo.

La pluralidad de papeles que tiene que ejercer la mujer, así como la necesidad de relacionarse, le lleva a tener una visión más global para la toma de decisiones, frente a la absorción del hombre por su trabajo.

La importancia que da la mujer a las relaciones conlleva, también, que se consideren receptoras de información para después hacerla llegar a los destinatarios, eso les crea la necesidad de compartir la información, favorece la visión más global y tiene efectos muy positivos en sus relaciones con el personal. El hombre suele pensar que el poseer información le da poder y le permite conseguir sus objetivos, frente a la mujer que piensa que el compartir esa información le facilitará la consecución de sus objetivos.

La mujer se caracteriza por ser más pragmática, por la perseverancia, cualidades que le proporcionan el éxito en los negocios.

La mujer, a pesar de que se le denomina el «sexo débil», es más resistente que el hombre. Resistente para afrontar contrariedades, vicisitudes, o el gran número de problemas que presenta el tener una responsabilidad.

Sin embargo, existen diversas opiniones que justifican la diversidad de las argumentaciones de los hombres y mujeres ante los diferentes problemas que se presentan en la empresa, no por su diferencia genética sino por su diferente evolución histórica. Ni la edad, ni la generación a la que se pertenezca, ni el sexo, son en sí mismas variables explicativas de las diferencias entre las personas. Hay que contemplar qué acontecimientos sociales, históricos, personales se ven reflejados en las diferencias de personalidad individuales.

En definitiva, hay que feminizar la empresa, no en el sentido peyorativo de la palabra, sino en el de incorporar las preocupaciones y puntos de vista de la mujer en la actuación de los directivos.

\section{Conclusiones}

La sociedad todavía pone trabas a la incorporación de la mujer a todos o a ciertos niveles del mercado laboral. Es verdad, que las últimas regulaciones 
laborales consideran la discriminación de la mujer y reaccionan apoyándola. Así, se han desarrollado normas laborales que apoyan la maternidad, la lactancia, el cuidado de los hijos menores de 6 años; se ha fomentado la contratación indefinida de mujeres en profesiones u oficios en los que se considera que la mujer está subrepresentada.

Pero el cambio definitivo no se logra concediendo ventajas económicas a las empresas. No se conseguirá la igualdad del hombre y la mujer en el mercado laboral si no se logra un cambio de los estereotipos existentes sobre la mujer.

Son las mujeres, con su esfuerzo y con su ejemplo, las que tienen que conseguir que la sociedad cambie y las acepte tal y como son, con su diversidad de papeles a ejercer; no quieren sentirse mutiladas, para lo cual además necesitarán la ayuda de todos los que les rodean. Tienen la responsabilidad social, mayor cuanto más alto sea el cargo que se ocupa, de cambiar los estereotipos existentes.

El cambio no sólo ha de promoverlo la mujer que trabaja fuera de casa, sino también aquellas mujeres que han optado por centrarse en los papeles de madre y esposa, la educación que se proporciona a los hijos no cae en saco roto, los cambios de mentalidad supondrán, en el futuro, cambios en la situación socioeconómica.

Es de resaltar el papel que la educación puede jugar en el necesario cambio de la sociedad, y eso es tarea de todos.

No hay que alegrarse de la buena suerte, porque la arbitrariedad de la discriminación en cualquier momento se puede volver en contra, y en ese momento se lamentará no haber luchado contra ella.

Esta sociedad cambiante exige una continua adaptación y flexibilidad, a la que la mujer, con sus valores tradicionales, puede aportar mucho. En la era de la información y de los medios de comunicación destaca la pérdida de la comunicación interpersonal, entorno en el que algunas de las aptitudes de la mujer pueden hacer mucho bien, en busca del equilibrio laboral y humano.

La mujer no se tiene que conformar con su destino o su entorno, tiene que luchar por obtener la formación necesaria para el desarrollo de su actividad.

Se debe saber mantener los valores tradicionales una vez que se inserta en la vorágine de la actividad laboral.

Por último, se debe mantener el equilibrio entre los tres papeles: madre, esposa y trabajadora, que se quieren ejercer, y que ello suponga no sólo una satisfacción personal, sino también la satisfacción de los que le rodean, de lo contrario no se habrá conseguido. 